\title{
Advanced and Versatile Real-Time Emulation Platform for Heterogeneous Radio Access Systems
}

\author{
Francisco Bernardo, Anna Umbert, Miguel López-Benítez, Nemanja Vučević \\ Department of Signal Theory and Communications \\ Universitat Politècnica de Catalunya (UPC) \\ Barcelona, Spain \\ \{fbernardo, annau, miguel.lopez, vucevic\}@tsc.upc.edu
}

\begin{abstract}
This paper is aimed at showing the capabilities of the real-time testbed for all-IP beyond $3 \mathrm{G}$ heterogeneous wireless networks that has been developed in the framework of the European IST AROMA project. The main objective of the AROMA testbed is to provide an advanced and realistic framework where the benefits of the algorithms developed within the AROMA project for both the radio access network and core network parts, as well as for the management of the end-to-edge quality of service, can be demonstrated. This advanced and versatile real-time emulation platform has been designed to enable an accurate and precise evaluation of Radio Resource Management/Common Radio Resource Management (RRM/CRRM) strategies and end-to-edge Quality of Service (QoS) management policies as well as specific protocols, services and commercial applications before bringing them to a real system. Its wide applicability and full potential has been extensively treated in previously published works. This paper describes a demonstration that shows a reduced and portable version of the testbed running on laptops to illustrate some of the capabilities of this advanced and powerful real-time emulation platform.
\end{abstract}

\section{Categories and Subject Descriptors}

I.6.3 [Simulation and Modeling]: Applications; I.6.7 [Simulation and Modeling]: Simulation Support Systems - environments; J.2 [Computer Applications]: Physical Sciences and Engineering - engineering.

\section{General Terms}

Measurement, Performance, Experimentation, Verification.

\section{Keywords}

All-IP; beyond 3G; heterogeneous wireless networks; real-time

\section{INTRODUCTION}

Permission to make digital or hard copies of all or part of this work for personal or classroom use is granted without fee provided that copies are not made or distributed for profit or commercial advantage and that copies bear this notice and the full citation on the first page. To copy otherwise, or republish, to post on servers or to redistribute to lists, requires prior specific permission and/or a fee.

PM2HW2N'08, October 31, 2008, Vancouver, BC, Canada. Copyright 2008 ACM 978-1-60558-239-9/08/10 ...\$5.00.
Trends in mobile communications are evolving towards the integration of different wireless access networks and technologies into heterogeneous infrastructures where the Internet Protocol (IP) technology is becoming the cornerstone around which such networks are converging. In this context, the concept of all-IP is commonly used to refer to those systems that provide IP-based multimedia services over IP-based transport in both the Radio Access Network (RAN) and the Core Network (CN) parts. This type of communication systems are facing the challenge of providing continuous and ubiquitous connectivity through different technologies while preserving the negotiated Quality of Service (QoS) level for the end-user during the entire session. In this scenario, one of the main challenges that heterogeneous wireless systems must overcome is the ability to guarantee the seamless interoperability and efficient management of the different RANs in order to provide the user with a suitable and consistent QoS level. To this end, efficient Radio Resource Management and Common Radio Resource Management (RRM/CRRM) strategies need to be developed. Moreover, the $\mathrm{CN}$ features need also to be taken into account and efficient QoS policies to coordinate the RAN and CN parts must be defined to provide the required endto-edge (e2e) QoS level.

To estimate the performance of such strategies and policies for mobile communication systems the use of simulation platforms is common within the research and industrial communities. Nevertheless, to conduct meaningful and appropriate studies, and to accurately assess the performance of innovative solutions before considering a prototype or full-scale deployment, the evaluation over realistic emulation platforms is becoming essential as a step forward toward the implementation in a real system. Real-time emulators allow reproducing realistic scenarios to test algorithms, policies, protocols, services and applications under realistic conditions, thus constituting a powerful tool for evaluating the QoS level experienced by the end-user.

In this context, the aim of the proposed demonstration is to present a sophisticated real-time testbed that has been developed in the framework of the AROMA project [1]. The objective of the AROMA project is to devise and assess a set of specific resource management strategies and algorithms for both the RAN and CN parts that guarantee the e2e QoS in the context of an all-IP heterogeneous wireless access network. The main objective of the AROMA testbed is to provide a framework where the benefits of the developed RRM/CRRM algorithms and the proposed QoS management techniques can be demonstrated. This live demon- 
stration will show a reduced and portable version of the testbed running on laptops to illustrate some of the capabilities of this advanced and powerful real-time emulation platform. Its wide applicability and full potential have been extensively treated in numerous previously published works [2]-[5].

\section{THE AROMA TESTBED}

\subsection{General Description}

The presented testbed allows the real-time emulation of an all-IP heterogeneous wireless access network that includes the UMTS Terrestrial Radio Access Network (UTRAN), GSM/EDGE Radio Access Network (GERAN), and Wireless Local Area Network (WLAN) as well as the corresponding common $\mathrm{CN}$ based on DiffServ technology and Multi-Protocol Label Switching (MPLS) [6]. The evaluation platform emulates, in real-time, the conditions that the behavior of the all-IP heterogeneous network, including the effect of other users, produces on the user under test (UUT) when making use of real multimedia IP-based applications such as videoconference, streaming services, or web browsing. Such approach allows testing real applications on an e2e basis on a complete all-IP heterogeneous network with RRM/CRRM algorithms and e2e QoS management policies. The AROMA testbed is therefore a powerful emulation platform that enables an accurate evaluation of advanced RRM/CRRM strategies and e2e QoS mechanisms as well as specific protocols, services and commercial applications before bringing them to a real system.

\subsection{Software and Hardware Platform}

The AROMA testbed is implemented with twenty off-the-shelf Personal Computers (PCs). Two of them run Windows operating system (the applications' PCs) and eighteen PCs run Linux operating system. This approach has been proven to be adequate for its capacity to assure appropriate levels of real-time management while guaranteeing a high degree of flexibility. The capacities provided by Linux operating system to interact at low level with the kernel offer the possibility to tune accurately the performance required by the testbed, especially in the issues related with the real-time execution and management. To implement real-time operation a very high computational power is required. These computational requirements are out of the scope of today's offthe-shelf PCs. Then, a cluster of PCs has been constructed to distribute the computational load throughout different processors. To this end, a software tool named Communications Manager (CM) [7] was designed and developed to make this distribution completely transparent.

Figure 1 shows all the entities and connections of the AROMA testbed. Black connections correspond to user data interfaces, whereas red and blue connections correspond to control plane interfaces. The UUT has at its disposal one stand-alone PC to run the application (applications' client), and one stand-alone PC is used to run the main functionalities associated to the User Equipment (UE). To test symmetric services as video-conference and to serve multimedia applications such as web-browsing or streaming, a correspondent node (applications' server) is run in a standalone PC. A graphical management and configuration tool named Advanced Graphical Management Tool (AGMT) has been developed to configure the initialization parameters, to control the execution flow, to collect logged data and to obtain and monitor statistics during the real-time execution of the testbed.

\subsubsection{Radio Access Network}

The three mentioned Radio Access Networks (RANs) are emulated using three PCs for UTRAN (two of which implement HSDPA and HSUPA real-time emulators), one PC for GERAN and one PC for WLAN. A Traffic Switch (TS) is used to establish different interconnection configurations between the UE and the IRs in the CN depending on the RAN the UUT is currently connected to. There is a software mapping of the RANs to each Ingress Router (IR) that is set at the beginning of the testbed's execution (e.g., UTRAN is said to be attached to IR1 while GERAN and WLAN to IR2).

In uplink, the TS (1) captures the UUT's IP application packets from the Client's PC, (2) stores them in a buffer, and (3) passes

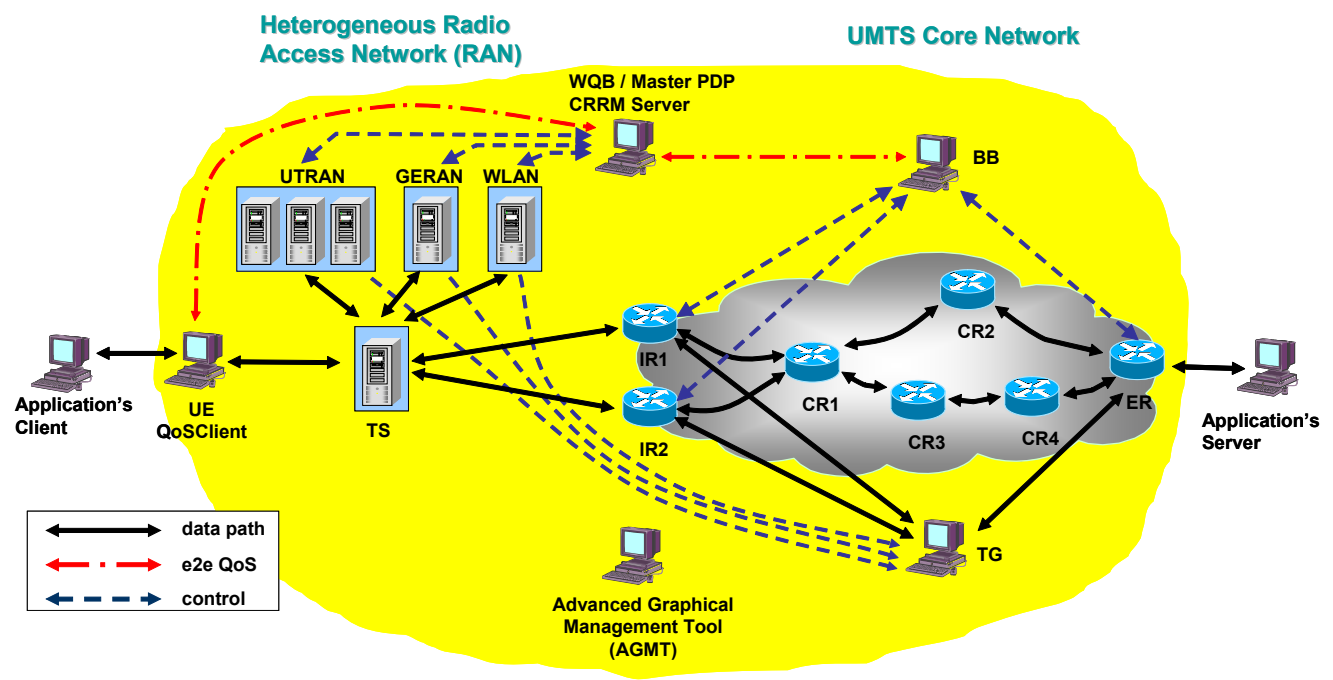

Figure 1. Entities and connections of the AROMA testbed. 
information about them (packet length, identifier) to the appropriate RAN where the UUT is connected to (to make the real-time emulation). After emulation, the RAN returns whether a specific packet should be forwarded or discharged from the buffer. In case that the packet is forwarded then the TS (4) re-injects it in the interface of the IR to which the RAN has been configured to be connected to. After that, the MPLS agents running on the CN routers forward the packet towards the ER following an MPLS LSP (Labeled Switched Path) that is selected according to the priority of the IP packet (i.e., the corresponding FEC (Forwarding Equivalence Class)). As an example, low priority packets may follow a longer path than the high priority ones, making traffic engineering possible. Finally, the packet is delivered to the Server PC. An analogous procedure is done in downlink.

The three Radio Access Network Emulators (RANEs) have been developed following carefully the corresponding standards. A highly detailed implementation of the specifications developed by the standardization bodies has been carried out, and several realistic and sophisticated models have also been implemented in these modules, which guarantee the accurate evaluation of the system performance. The three RANEs have been designed to cope with the following goals and requirements:

- Support for live users as well as fully emulated users, up to several thousands depending on scenario and traffic configuration. Traffic of all of the users is processed by RAN emulators. Therefore, the UUT behaves as any other user in the system.

- Emulation of the transmission chain between the mobile terminal and the radio network controller. Different functions performed at each level of the protocol stack have been precisely modeled in accordance to the specifications.

- Execution of RRM functions (admission control, congestion control, radio resource allocation, handover management, outer and inner loop power control and transmission parameters management) as well as support for CRRM capabilities.

- Support for different communication scenarios, according to the requirements and visions of the four mobile operators that participated in the AROMA project.

- Emulation of all-IP RAN. A model for emulating the effects and impairments of the IP-based transport in the RAN has been implemented. The envisaged IP-RAN emulation model takes into account losses in the transport network, obtained from non-real-time simulations. These loss statistics obtained from off-line simulations are used to determine, for different scenarios, the probability that an IP packet is lost in the IP-RAN transport network, which is used to decide in real-time whether a packet is discarded due to IP transport impairments.

\subsubsection{Core Network}

The $\mathrm{CN}$ is not emulated. It has been built using seven Linux PCs instead, which act as routers of a DiffServ domain with MPLS forwarding. Three PCs serve as edge routers: two of them are Ingress Routers (IRs) while the other PC is an Egress Router (ER). The other four PCs in the CN, identified as Core Routers
(CR), are used to connect the edge routers providing different paths between them. These routers work using the communication protocol stack of the Linux operating system enhanced with MPLS support.

Inside the $\mathrm{CN}$, packets are forwarded based on their FEC (Forwarding Equivalence Class), which determines and makes the flow to follow a pre-established LSP (Labeled Switched Path). With this, different type of traffic may experience different QoS. Packets are marked in the Ingress Routers, with the correct MPLS label corresponding FEC they belong to.

Figure 1 shows a (slightly unbalanced) fish model that demonstrates how routes can be set up in the $\mathrm{CN}$. This model can be used to simulate and model traffic flows for setting up applications such as traffic engineering (TE). Conventional IP routing would most likely set up a route from CR1 to CR2 to ER1 for traffic that is being sent from a host in the RAN to a host in the backbone network, since it is the shortest path. The routing protocols running on these nodes would create forwarding information bases that would direct the packets along the short path route. However, with MPLS, it can be used a TE application, a constraint-based signaling and label distribution (such as RSVP-TE or CR-LDP) to set up a LSP tunnel from CR1 to CR3 to CR4 to ER1 as an alternative route.

The link between the emulated Access Network (AN) and the CN is an Interworking PC that captures the emulated traffic and injects it in the CN. The traffic injection differentiation, to the IR1 or IR2, depends on the network source of the traffic (e.g., UTRAN, GERAN, etc.) in the AN.

For the emulated users passing through the testbed there is a PC called Traffic Generator (TG) that is in charge of generating real IP traffic to load the $\mathrm{CN}$ in accordance to the traffic amount that active users generate in the system. Obviously, generation of this traffic is coordinated with the traffic emulated in the radio part.

\subsubsection{End-to-Edge QoS Management Framework}

The AROMA testbed constitutes a realistic framework to test different e2e QoS strategies and evaluate the QoS level provided when real client-server IP based applications are executed in the edges of the testbed. The perceived QoS will be measured once the real IP packets of the UUT have passed through the testbed and have been impaired according to the Radio and Core network status. Moreover, this framework allows the testing of particular implementation of the QoS entities which may be important for operators before applying these implementations to real networks.

The e2e QoS management architecture is composed of the QoSClient, the Wireless QoS Broker (WQB) in the RAN part, and the Bandwidth Broker (BB) in the CN part, as it can be seen in Figure 1. The QoSClient is the entity that provides an interface from which the UUT can activate, deactivate and modify the sessions with QoS guarantees. Three service classes are available for the UUT: conversational, streaming and interactive. The BB is the entity that handles the QoS management in the $\mathrm{CN}$, by configuring the proper DiffServ filters in the IRs and ERs and establishing MPLS tunnels for the UUT between these routers. Depending on the UUT's traffic class the MPLS tunnel is established through CR2 or, alternatively, through CR3 and CR4 where a higher delay is expected. Additionally, the WQB handles the QoS in the radio part, and tightly interacts with the Common Radio Resource 
Management (CRRM) entity, which is in charge of functions such as Radio Access Technology (RAT) selection and Vertical Handover (VHO) procedures. The QoS requested by the UUT is preserved along the RAN and $\mathrm{CN}$ domains due to the e2e negotiation that WQB and BB perform during real-time execution.

In the testbed, the e2e QoS support is enabled by making interaction between these entities where WQB leads the QoS negotiation. It receives a QoS request from the QoS Client, and it consults CRRM and BB about their QoS capabilities for the acceptance of a new session. Then WQB finally takes decisions on session establishment (or modification) based on the information provided by the CRRM and $\mathrm{BB}$. In fact, the information that CRRM generates during the e2e QoS negotiation actually bases on Admission Control and RAT Selection algorithms, whereas the $\mathrm{BB}$ decision is based on its proper Connection Admission Control (CAC) algorithms.

Different mechanisms for the QoS negotiation between QoS entities have been implemented to allow the UUT to initiate a session with QoS guarantees or seamlessly renegotiate the QoS during the session. Logically, this framework requires the development of a proper interface between the mentioned entities. In the testbed, a three-handshake signaling procedure has been developed with that purpose. Any negotiation between two entities is done by means of the exchange of three messages named REQuest (REQ), DECision (DEC) and RePorT (RPT). Any negotiation interaction between the QoS entities is initiated by a REQ message which encapsulates the session id, the flow attributes (source and destination IP addresses and ports), the performance attributes (including the requested QoS in terms of throughput, packet loss, delays, and DiffServ code point, etc.) and the conformance attributes (needed for the traffic shaping in the ingress routers). Then, the entity that receives the REQ replies with a DEC message indicating whether the requested QoS can be supported or not. Finally, the negotiation is closed with a RPT message that originates the enforcement of the negotiated QoS if the negotiation was successful.

\subsubsection{Applications}

The QoS perception has been defined as one of the goals in the AROMA testbed. The general objective is to make the quality measurements with several applications in order to test the QoS perceived by the user under test (UUT) in different network (endto-end) conditions. One of the benefits of the testbed is that real IP-based multimedia applications can be installed and tested in terms perceived QoS within the platform. This property is one of the differential features of the testbed when compared with conventional simulators.

The primary requirement for the applications that should be used in perceptual QoS evaluation is to be widely available. Both commercial and open source applications have been considered. Depending on the specific behavior in the network that is tried to be evaluated, some of the applications that may be used are given in Table 1.

To evaluate the perceived QoS, the application needs to be captured on the user's side. The modified (degraded) multimedia contents are compared to the reference contents (originals). The applications used should respect the recommendations of QoS metrics [8][9] and be in accordance with the input file types (audio, speech or video).
Table 1 Applications installed in the testbed

\begin{tabular}{|c|c|c|c|}
\hline & \multicolumn{2}{|c|}{ End to End Application } & \multirow{2}{*}{$\begin{array}{c}\text { Capturing } \\
\text { Application }\end{array}$} \\
\hline $\begin{array}{l}\text { End to End } \\
\text { Service }\end{array}$ & Server & Client & \\
\hline \multirow[t]{2}{*}{ Video Streaming } & \multirow{2}{*}{$\begin{array}{l}\text { Darwin } \\
\text { Streaming } \\
\text { Server }\end{array}$} & $\begin{array}{c}\text { QuickTime } \\
\text { Pro }\end{array}$ & \multirow{2}{*}{$\begin{array}{l}\text { Camtasia } \\
\text { Studio Re- } \\
\text { corder }\end{array}$} \\
\hline & & $\begin{array}{c}\text { Video Lan } \\
\text { Client }\end{array}$ & \\
\hline \multirow[t]{2}{*}{ Audioconference } & $\begin{array}{c}\text { Robust Audio } \\
\text { Tool }\end{array}$ & $\begin{array}{c}\text { Robust } \\
\text { Audio Tool }\end{array}$ & \multirow{2}{*}{$\begin{array}{l}\text { Microsoft } \\
\text { Sound Re- } \\
\text { corder }\end{array}$} \\
\hline & NetMeeting & NetMeeting & \\
\hline Videoconference & $\begin{array}{c}\text { VIC video } \\
\text { conference tool }\end{array}$ & $\begin{array}{l}\text { VIC video } \\
\text { conference } \\
\text { tool }\end{array}$ & $\begin{array}{l}\text { Camtasia } \\
\text { Studio Re- } \\
\text { corder }\end{array}$ \\
\hline
\end{tabular}

\section{APPLICABILITY}

As it has been said, one of the key objectives of the presented testbed is to enable testing the e2e QoS performance and to evaluate, in real-time, the effects that the implemented e2e QoS management algorithms have over the user's perception when using different QoS classes.

In particular we stress studies addressed to:

- $\quad$ Evaluate how different network procedures impact over the user perceived QoS of real multimedia applications, like Net-Meeting ${ }^{\circledR}$ and QuickTime ${ }^{\circledR}$.

- Evaluate RRM/CRRM functions, such as admission control or RAT selection algorithms, in a realistic scenario.

- Evaluate subsystems and protocols that rely on real implementations.

- $\quad$ Analyze how the dynamic Label Switched Path (LSP) management through the $\mathrm{CN}$ impacts on the e2e performance.

- $\quad$ Test mechanisms such as IP fast handover with mobility support.

- $\quad$ Evaluate e2e QoS signaling procedures and policies developed within the testbed and how they impact on the user's QoS preservation.

- Test the performance of innovative RAT such as HSPA under realistic conditions and traffic patterns.

This demonstration is aimed at showing a reduced and portable version of the real-time testbed for all-IP beyond $3 \mathrm{G}$ heterogeneous wireless networks that has been developed in the framework of the European IST AROMA project. Since the real AROMA testbed is composed of twenty PCs (see Figure 2), this demonstration will show a reduced and portable version of the testbed running on two laptops (see Figure 3). This reduced version with limited computational capabilities will however suffice to illustrate many functionalities of the AROMA testbed as well as sev- 


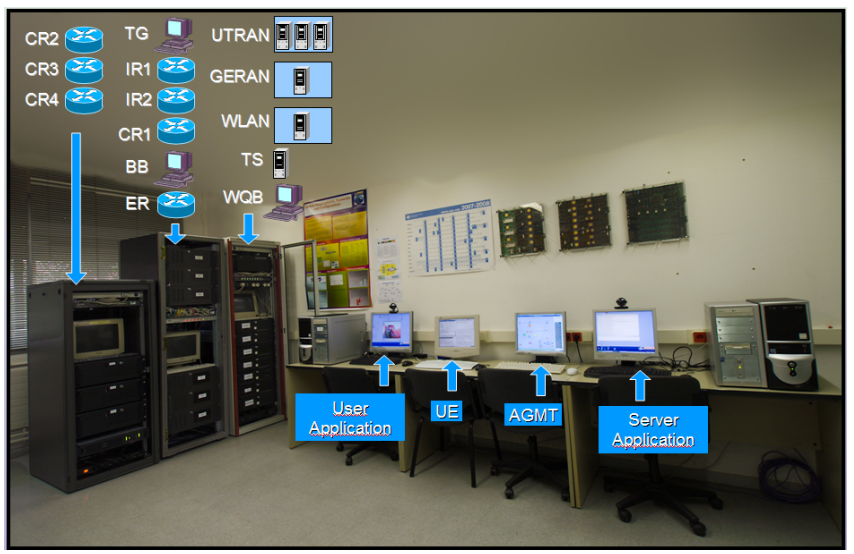

Figure 2. The AROMA testbed at UPC's premises.

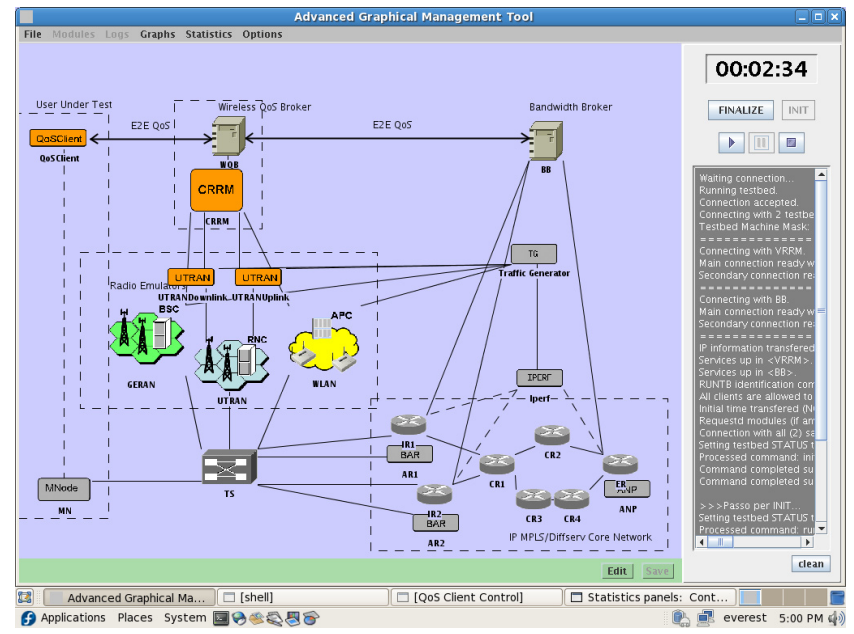

Figure 4. AGMT graphical interface.

eral types of studies that can be conducted with this advanced and powerful real-time emulation platform.

The two-laptop version runs an operational version of the testbed that also works in real-time. This demonstration version will allow us to show the graphical interface of the testbed and all its functionalities (see Figures 4 and 5) such as configuration parameters for the different modules that integrate the testbed, realtime monitoring of testbed's parameters and statistics, tools for saving and post-processing results, off-line replaying the system behavior, etc. Moreover, some case studies illustrating the testbed ability in evaluating algorithms and policies, and providing a better understanding of system performance will also be shown.

A third laptop will be used to show some screenshots of more complex and sophisticated case studies that can be carried out with the real AROMA testbed. Two demonstration videos of the AROMA testbed (see AROMA's web page [1], "Demonstration Videos" section) will also be displayed during the demonstration.

Three posters (A0 size, $841 \times 1189 \mathrm{~mm}$ ) providing a general overview of the testbed and a detailed description of the RAN and $\mathrm{CN}$ parts will also be shown during the demonstration. Additionally, demonstration CDs with information related to the AROMA project (project description, deliverables, publications, white pa-

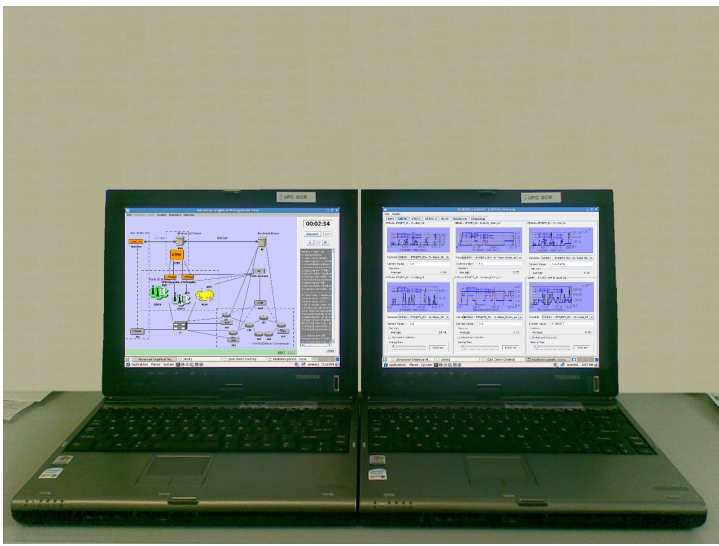

Figure 3. Demonstration version of the AROMA testbed.

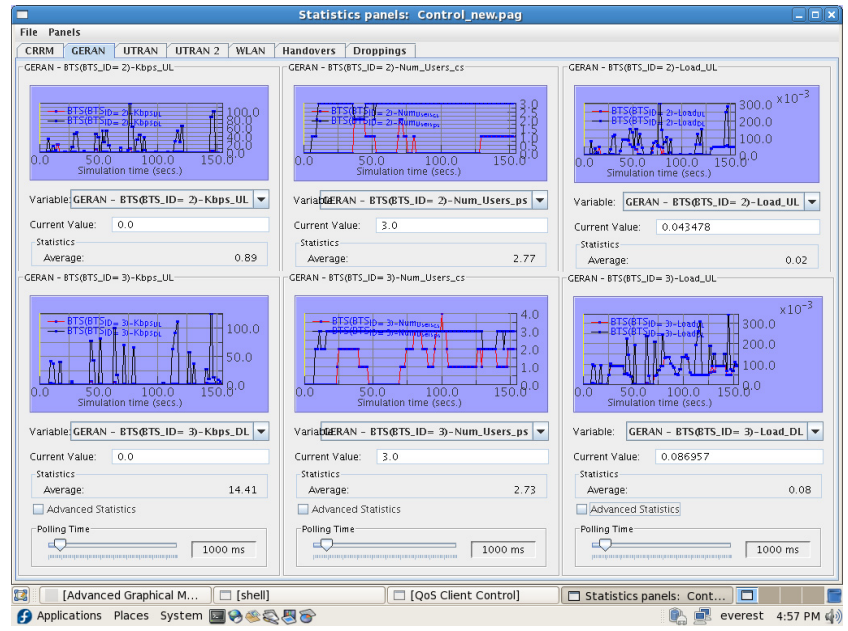

Figure 5. Panel showing some statistics in real-time.

pers, demonstration videos, etc.) will be offered to the public attending the Demos/Tools session.

\section{ACKNOWLEDGMENTS}

This work has been performed in the framework of the IST AROMA Project, and has been supported by the Spanish Research Council under COGNOS grant (ref. TEC2007-60985).

\section{REFERENCES}

[1] AROMA Project (Advanced Resource Management Solutions for Future All IP Heterogeneous Mobile Radio Environments), 6th Framework Programme of the European Community, http://www.aroma-ist.upc.edu.

[2] Umbert, A., López-Benítez, M., Bernardo, F., Vučević, N., Azevedo, R., Oliveira, A., "The real-time AROMA testbed for all-IP heterogeneous wireless access networks", 4th International Conference on Testbeds and Research Infrastructures for the Development of Networks \& Communities (TRIDENTCOM 2008), March 2008, p. 10.

[3] Bernardo, F., Vučević, N., Umbert, A., López-Benítez, M., "Quality of experience evaluation under QoS-aware mobility 
mechanisms", in Proceedings of European Wireless 2008 (EW 2008), Prague, Czech Republic, June 2008, p. 7.

[4] Vučević, N., Bernardo, F., Umbert, A., López-Benítez, M., "End-to-edge QoS across heterogeneous wireless and wired domains", in Proceedings of the Fifth International ICST Conference on Heterogeneous Networking for Quality, Reliability, Security and Robustness (QShine 2008), Hong Kong, China, July 2008, p. 7.

[5] López-Benítez, M., Bernardo, F., Vučević, N., Umbert, A., "Real-time HSPA emulator for end-to-edge QoS evaluation in all-IP beyond 3G heterogeneous wireless networks", in Proceedings of the $1^{\text {st }}$ International Workshop on the Evaluation of Quality of Service through Simulation in the Future Internet (QoSim 2008), Marseille, France), March 2008, p. 12 .
[6] Rosen E., et al., "Multiprotocol Label Switching Architecture”, IETF RFC 3031, January 2001.

[7] Revés X., et al, "Implementation of a Real-time UMTS Testbed in a PC Network", ACIS 3rd SNPD, June 2002, pp. 345-352.

[8] Muntean, G.-M., Perry, P., Murphy, L., "Objective and Subjective Evaluation of QOAS Video Streaming over Broadband Networks", eTransactions on Network and Service Management, vol. 2 , no. 1, November 2005.

[9] ITU-T Recommendation J.144 Revised, "Objective perceptual video quality measurement techniques for digital cable television in the presence of a full reference", March 2004. 